\title{
ÁREAS VERDES E ISLA DE CALOR URBANA
}

\section{GREEN AREAS AND URBAN HEAT ISLAND}

\section{ARELLANO, Blanca}

Universidad Politécnica de Catalunya (UPC)

Departamento de Tecnología de la Arquitectura (TA), Centro de Política de Suelo y Valoraciones (CPSV) Profesora Asociada y Personal de Investigación

Av. Diagonal 649, 08028 Barcelona, España

Correo electrónico: blanca.arellano@upc.edu

Teléfono: +34 934054383

\section{ROCA, Josep}

Universidad Politécnica de Catalunya (UPC)

Departamento de Tecnología de la Arquitectura (TA), Centro de Política de Suelo y Valoraciones (CPSV) Catedrático de Universidad

Av. Diagonal 649, 08028. Barcelona, España

Correo electrónico: josep.roca@upc.edu

Teléfono: +34 934016396

Palabras Clave: Isla de Calor Urbana; Cambio climático; Islas de Frío; Planeamiento; Morfología Urbana; Espacio Público

Key words: Urban Heat Island; Climate change; Islands of Cool; Planning; Urban Morphology; Open Space

\section{Resumen}

La temperatura de la superficie del suelo (LST) juega un papel clave en la generación de la isla de calor urbano (ICU), representando un factor determinante de la radiación de la superficie terrestre en el intercambio de energía y el control de la distribución del calor con la atmósfera. La composición de las cubiertas de suelo es uno de los principales factores que influyen en la LST. La literatura ha destacado que los diferentes tipos de materiales de cobertura del suelo, su albedo y rugosidad, el grado de impermeabilidad, así como la calidad y cantidad de vegetación, pueden tener un impacto significativo en la generación de la ICU.

El objetivo general de esta ponencia es investigar si el diseño urbano, y muy concretamente el de las zonas verdes, puede, a la pequeña escala, mitigar la Isla de Calor Urbano. La pregunta planteada en la investigación es si la morfología del espacio público, especialmente la forma y la zonificación de áreas verdes y espacios abiertos, influye en la temperatura de la superficie del suelo (LST) y del aire (LSAT) y, por lo tanto, en el ICU. La hipótesis de trabajo es que la morfología del espacio público juega un papel clave para controlar la ICU. Por lo tanto, un diseño adecuado de espacios abiertos puede ayudar a aumentar la resiliencia de las ciudades al Cambio Climático (CC).

Para probar la hipótesis, se eligieron dos estudios de caso en la ciudad de Sant Cugat del Vallès (en el área metropolitana de Barcelona): dos desarrollos urbanos realizados en los años 80 muy cercanos el uno del otro (Parc Central y Coll Favà). Ambos con unas características similares, pero con un diseño de espacio público claramente diferenciado; mientras que en 
Parc Central, el área construida se estructura alrededor del espacio abierto, en Coll Favà, la gran mayoría de los espacios abiertos se ubican periféricamente, rodeando las áreas construidas. La metodología de investigación consistió en: a) estudiar los parámetros urbanos y climáticos de las áreas seleccionadas; b) analizar la distribución espacial del LST utilizando tecnologías de detección remota (Landsat 8); c) obtener LST y LSAT a través del trabajo de campo, durante el día y la noche; y d) construir un modelo de temperatura de la superficie y del aire en función de los diferentes tipos de cobertura del suelo, combinando datos de teledetección y mediciones in situ, para cada una de las áreas de análisis.

\section{Abstract}

The Land Surface Temperature (LST) plays a key role in the generation of the Urban Heat Island $(\mathrm{UHI})$, representing a determining factor of the radiation of the surface and the exchange of energy and control the distribution of heat between the surface and the atmosphere. The composition of the land covers is one of the main factors that influence the LST. The literature have highlighted that the different types of land cover materials, their albedo and roughness, degree of impervious as well as the quality and quantity of vegetation, can have a significant impact on the generation of the UHI.

The overall objective of the paper is to investigate if urban design, on a detail scale, can mitigate the Urban Heat Island. The question is if the morphology of public space, especially the form and zoning of green areas and open spaces, influence surface (LST) and air (LSAT) temperatures and therefore in the UHI. The working hypothesis is that the morphology of public space plays a key role to control UHI. Thus, an adequate design of open spaces can help to increase the resilience of cities to Climate Change (CC).

To prove the hypothesis, two case studies were chosen. The case studies are two urban expansion areas of city of Sant Cugat del Vallès (in the metropolitan area of Barcelona), the first one, called Parc Central and the second one, Coll Favà. Both have similar characteristics, but with a clearly differentiated public space design; while in Parc Central built-up area is structured around the open space, in Coll Favà, the vast majority of open spaces are located peripherally, surrounding the built-up areas. The research methodology consisted in: a) studying the urban and climatic parameters of selected areas; b) analyzing the spatial distribution of the LST using remote sensing technologies (Landsat 8); c) obtaining LST and LSAT through field work, during day and night time; and d) constructing a model of surface and air temperatures as a function of the different types of land cover, combining Remote Sensed data and in situ measurements, for each of the areas of analysis.

\section{Introducción}

"Warming of the climate system is unequivocal, and since the 1950s, many of the observed changes are unprecedented over decades to millennia. The atmosphere and ocean have warmed, the amounts of snow and ice have diminished, and sea level has risen." (IPCC 20141, página 30). "Each of the last three decades has been successively warmer at the Earth's surface than any preceding decade since 1850 . The period from 1983 to 2012 was very likely the warmest 30-year period of the last 800 years in the Northern Hemisphere, where such assessment is possible (high confidence) and likely the warmest 30-year period of the last 1400 years (medium confidence)" (IPCC 2014¹, página 40). Este aumento de la temperatura se distribuye de forma general en todo el planeta, siendo más pronunciado en las latitudes situadas más al norte. Las regiones terrestres (fig. 1) se vienen calentando de forma más 
rápida que los océanos. Del mismo modo puede afirmarse que la variación de las concentraciones de gases de efecto invernadero (GEI) en la atmósfera así como las variaciones de radiación en las coberturas de suelo y en la radiación solar, alteran el balance energético del sistema climático. En este sentido, el origen antropogénico de los cambios observados ${ }^{2}$ parece hoy un hecho igualmente incontrovertible. Las emisiones globales de GEI debidas a los efectos de las actividades humanas han aumentado en un $70 \%$ entre 1970 y 2004. El resultado de los diferentes modelos de la evolución de las temperaturas de la superficie terrestre, muestra la prominencia del origen de forzamiento antropogénico, con respecto al de naturaleza natural.

Figura 1. Evolución de las temperaturas (forzamiento natural y antropogénico)

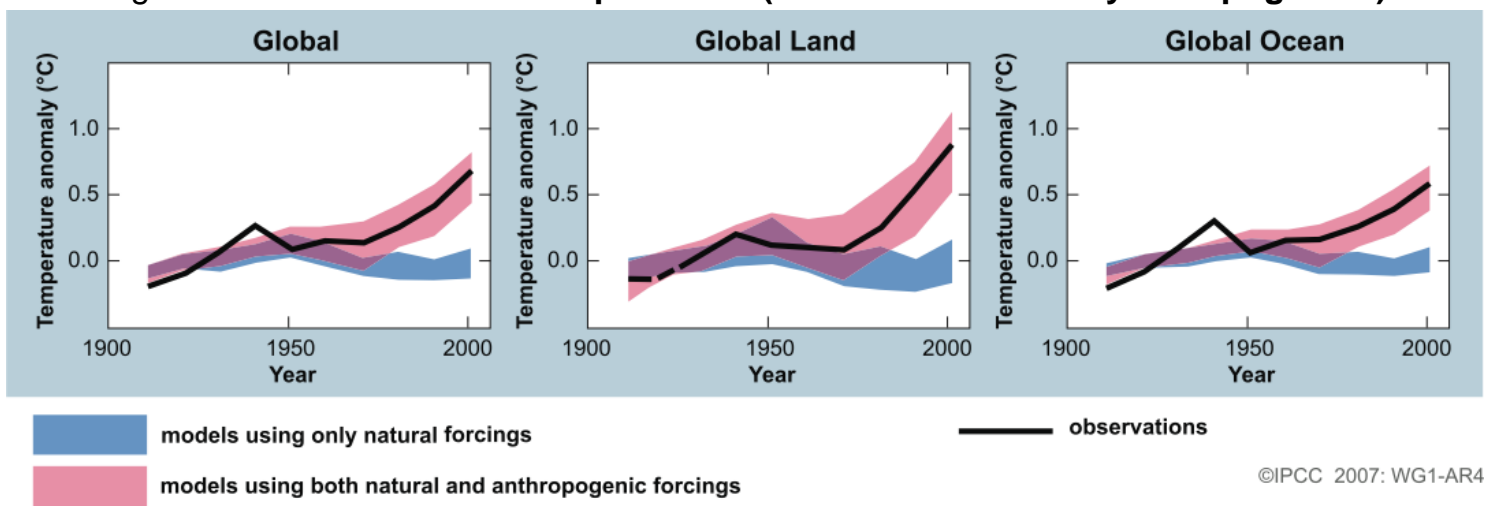

Fuente: IPCC 2007

Existe un elevado consenso científico acerca del protagonismo de las ciudades en el Cambio Climático. Según el Center for Human Settlements (UN-HABITAT), las ciudades son responsables del $75 \%$ del consumo mundial de energía, y del $80 \%$ de las emisiones de GEl. La contribución de la urbanización al cambio climático es de doble naturaleza: por un lado, por la generación urbana de GEI, factor que contribuye de manera decisiva al calentamiento global del planeta; por otro lado, por la radiación generada por la superficie del suelo urbanizado, que determina una transferencia acusada de calor sensible en función al tipo de cubierta urbana, así como a su reducido grado de humedad.

Si bien el clima urbano depende esencialmente de factores globales y regionales, factores locales y de microescala (como las diferentes características de la estructura urbana, la topografía y la superficie de las cubiertas de suelo, la vegetación, el calor antropogénico generado por el metabolismo urbano, ...) pueden modificar el clima local a escala urbana.

Existen diferencias significativas entre el clima de las áreas urbanas y rurales: la isla de calor urbano (ICU) describe la influencia de las superficies urbanas en los patrones de temperatura de las áreas urbanas en comparación con las áreas circundantes. La superficie sellada del suelo y los materiales artificiales (especialmente el asfalto y el hormigón) que se usan generalmente en áreas urbanas son una de las principales causas.

Los efectos de la Isa de Calor Urbana se manifiestan en diferentes escalas. Se pueden distinguir dos tipos de ICU: la "isla de calor de la canopia urbana" y la "isla de calor del límite urbano". El primero depende de la rugosidad del suelo generado por los edificios y el dosel de

Citación: ARELLANO, B. y ROCA, J. Áreas verdes e Isla de Calor Urbana. En: Libro de proceedings, CTV 2018. XII Congreso Internacional Ciudad y Territorio Virtual. "Ciudades y Territorios Inteligentes". Mendoza, 5-7 septiembre 2018. Barcelona: CPSV, 2018, p. 417-432. 
los árboles, con un límite superior ubicado justo por encima del nivel de los techos de los edificios. En esta capa, el flujo de aire y los intercambios de energía se rigen por procesos de microescala que dependen de las características específicas de la superficie. El segundo está situado sobre el primero, con un límite inferior sujeto a la influencia de la superficie urbana en su conjunto. En la capa límite urbana, que es la parte de la capa límite atmosférica sobre los espacios urbanizados cuyas características se ven afectadas por la presencia de la ciudad, la ICU opera de una manera diferente, representando un fenómeno de mesoescala local. Es decir, de un fenómeno controlado por procesos que operan en una mayor escala espacial y temporal.

Existe un amplio consenso de que la temperatura de la superficie del suelo (LST) juega un papel clave en la generación de la Isla de Calor Urbano, lo que representa un factor determinante en términos de la radiación generada por la superficie, así como del intercambio de energía, además de controlar la distribución de calor entre la superficie y la atmósfera ${ }^{3}$.

La composición de las cubiertas de suelo es uno de los principales factores que influyen en la LST. La literatura especializada ha enfatizado que el área edificada, la superficie permeable y provista de vegetación, así como la superficie impermeable (áreas pavimentadas y construidas) tienen un impacto significativo en la generación de la ICU4 . En este sentido, la reducción de la vegetación afecta no solo al aumento de la LST, sino también a la reducción de la precipitación y la evapotranspiración. La relación entre el LST y el Normalized Difference Vegetation Index (NDVI) está especialmente bien documentada.

Por su parte, la teledetección proporciona información espacialmente continua que permite obtener el LST en escalas que van de decenas de metros (Landsat) a kilómetros (MODIS). Los satélites proporcionan datos relevantes acerca de las características y los usos del suelo, los cuales permiten cuantificar la ICU por medio de diversos indicadores ${ }^{5}$. La teledetección ha hecho posible el estudio generalizado del LST y, en consecuencia, del UHI a escala local y regional.

Si bien la LST se puede medir fácilmente mediante detección remota, la ICU se ha estudiado principalmente a través de la temperatura del aire en la capa superficial, generalmente a una altura de $2 \mathrm{~m}$ sobre el suelo (temperatura de la superficie del aire terrestre, LSAT). La temperatura del aire, medida en las estaciones meteorológicas, es una de las observaciones registradas con mayor frecuencia, con gran precisión y resolución temporal.

La temperatura del aire, a pesar de su menor relevancia climática en relación con la LST, tiene un papel esencial en la escala del confort humano. Es la temperatura que perciben las personas, y en relación con la cual el intercambio de energía interactúa con el medio ambiente urbano. Sin embargo, generalmente hay pocas estaciones meteorológicas, lo que en términos generales significa que los datos obtenidos de las estaciones no representan de manera eficiente la variación espacial de la temperatura del aire a nivel intramunicipal o de barrio o tejido urbano. Por ejemplo, en el RMB (Región Metropolitana de Barcelona) solo hay 33 estaciones meteorológicas.

Citación: ARELLANO, B. y ROCA, J. Áreas verdes e Isla de Calor Urbana. En: Libro de proceedings, CTV 2018. XII Congreso Internacional Ciudad y Territorio Virtual. "Ciudades y Territorios Inteligentes". Mendoza, 5-7 septiembre 2018. Barcelona: CPSV, 2018, p. 417-432. 
Figura 2. LST y LSAT (día y noche)

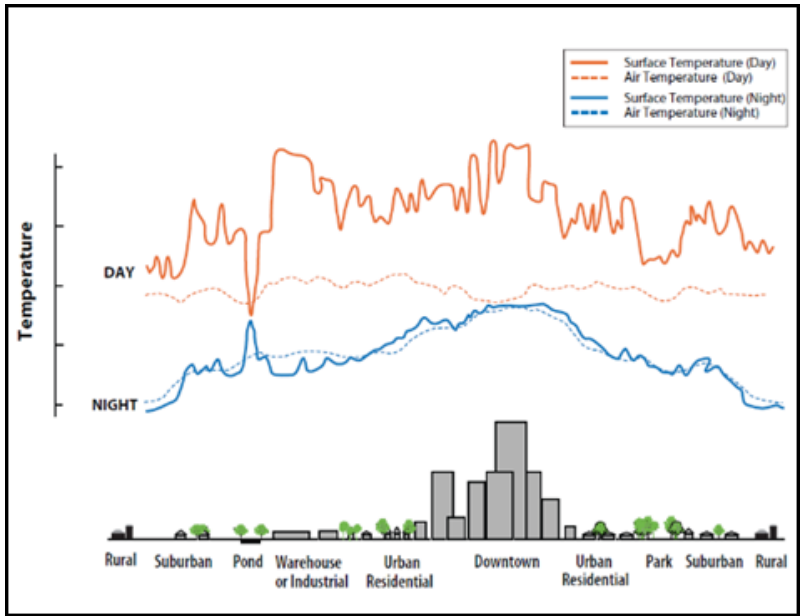

Fuente: Environmental Protection Agency $(2008)^{6}$

Especialmente relevantes, para el control de la Isla de Calor Urbana, son las áreas verdes urbanas. Debido a su abundante vegetación y su superficie permeable, los parques presentan un comportamiento diferente al resto de los espacios urbanos. En este sentido se ha estudiado la posibilidad de utilizar los parques como estrategia para reducir la ICU de las ciudades. La literatura especializada reconoce que la vegetación es uno de los principales componentes del espacio urbano que causa el enfriamiento.

Cuando se produce una disminución en las temperaturas del suelo y del aire debido a la abundancia de áreas verdes, se dice que existe una "Isla de Frio Urbano"7, que consiste en un efecto de enfriamiento dentro del área verde y en sus alrededores. Su influencia se manifiesta tanto en la LST como en la LSAT, y sus efectos ocurren especialmente de día, pero también de noche, aunque la extensión de su extensión e intensidad del enfriamiento depende de las características del espacio verde y la configuración urbana de su entorno 7,8 .

\section{Objetivo de la investigación}

El objetivo general de la investigación es estudiar, utilizando técnicas de detección remota, así como mediciones "in situ", cómo afecta el diseño urbano en la generación de la Isla de Calor Urbana, así como el microclima urbano en general. Específicamente, esta ponencia busca esclarecer si el diseño de áreas verdes puede mitigar la ICU.

El caso de estudio es la Región Metropolitana de Barcelona (figura 3), que incluye 164 municipios, con una población de 4,7 millones de habitantes y una extensión de 3.200 kilómetros cuadrados. A dicha escala metropolitana, se estudiará la configuración espacial de la Isla de Calor Urbana. Mientras que, a escala local, se analizará cómo la morfología del espacio público, especialmente de las áreas verdes y espacios abiertos, afecta a las temperaturas de superficie (LST) y de aire (LSAT), determinando las características de la ICU.

Citación: ARELLANO, B. y ROCA, J. Áreas verdes e Isla de Calor Urbana. En: Libro de proceedings, CTV 2018. XII Congreso Internacional Ciudad y Territorio Virtual. "Ciudades y Territorios Inteligentes". Mendoza, 5-7 septiembre 2018. Barcelona: CPSV, 2018, p. 417-432. 
Figura 3. Región Metropolitana de Barcelona

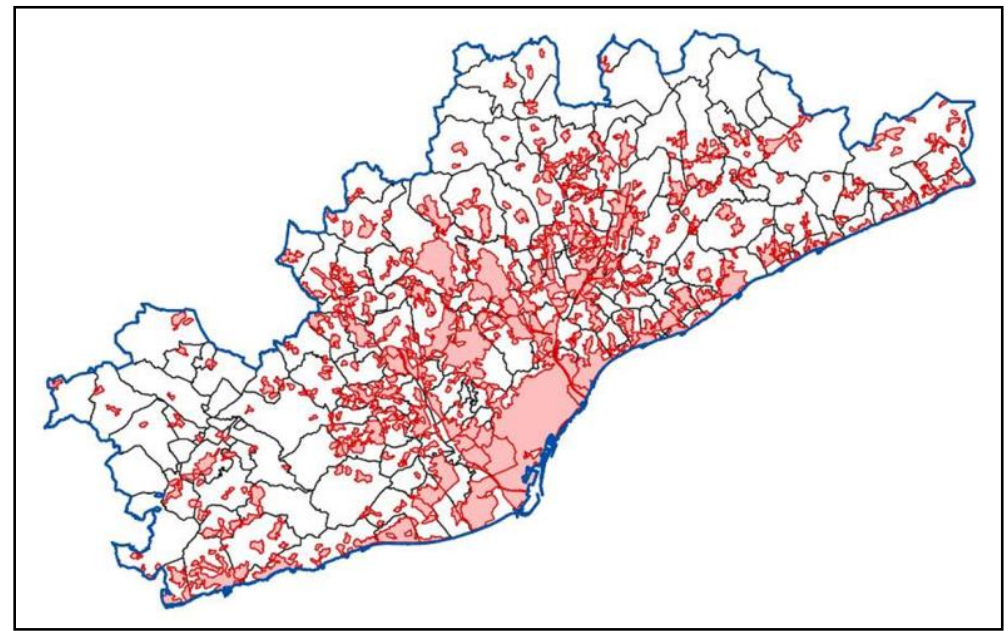

Fuente: Elaboración propia

Se analizarán y compararán dos estudios de caso en el municipio de Sant Cugat del Vallés (SCV, figura 4): las urbanizaciones Parc Central (PC) y Coll Favà (CF), que tienen características urbanas similares, pero que se caracterizan por disponer de un diseño urbano claramente diferenciado, especialmente en su espacio público. Los edificios en Parc Central están construidos alrededor de espacios abiertos, mientras que en Coll Favà, la gran mayoría de los espacios abiertos están ubicados periféricamente, rodeando los edificios.

Figura 4. Casos de Estudio

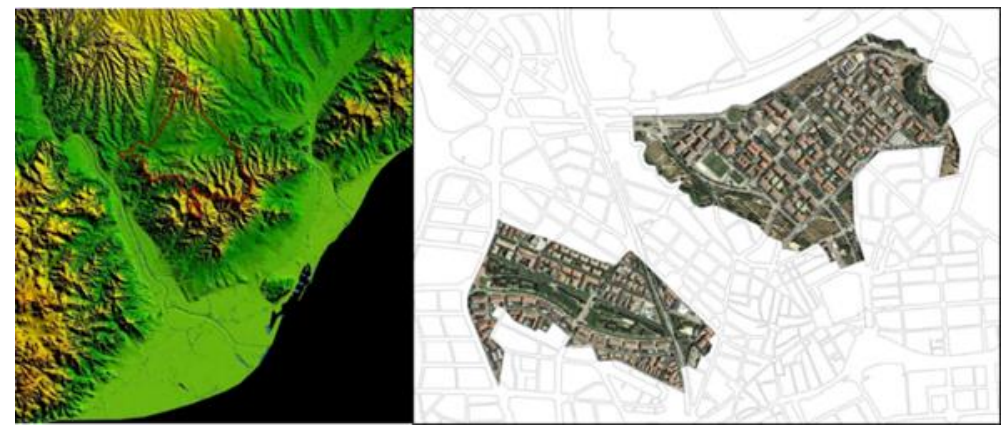

Fuente: Elaboración propia

La hipótesis de la investigación consiste en intentar demostrar que la morfología del espacio público es un elemento significativo en la configuración de la Isla de Calor Urbana. En particular, el diseño del espacio público, su morfología y la calidad de su vegetación determinan el grado de enfriamiento de las áreas verdes urbanas, delimitando la extensión e intensidad de la Isla de Frio Urbano.

\section{Metodología}

La metodología utilizada en este documento combina el uso de tecnologías de teledetección, de resolución media (30 metros/píxel), con la toma de mediciones "in situ". La combinación de ambos enfoques, la recolección remota y de datos "in situ", permite validar de manera más 
robusta las hipótesis de investigación relacionadas con el papel clave desempeñado por el diseño del espacio público, y especialmente de las áreas verdes, en la regulación de la ICU.

Específicamente, la metodología de investigación se desarrolla en los siguientes pasos:

1. Análisis de teledetección

a. La LST diurna se obtendrá a partir de LANDSAT 8, con una escala de resolución de 30x30 metros / píxel. La medición de la LST resultante permitirá una comparación inicial entre los casos de estudio (Parc Central y Coll Favà, ubicado en Sant Cugat del Vallès), evaluando cuál de los dos desarrollos muestra un efecto menos pronunciado del UHI.

b. La LST nocturna se obtendrá a partir de MODIS, a $1 \mathrm{~km} 2$ por píxel. Debido a la baja resolución de MODIS, los resultados de la noche LST no permiten un análisis detallado a nivel local. Sin embargo, la LST nocturna obtenida a través de MODIS nos permite conocer la configuración del UHI nocturna a escala metropolitana.

c. Las temperaturas diurna y nocturna, obtenidas por teledetección, permiten realizar una aproximación detallada de la UHI.

d. La información obtenida a través de los satélites permite conocer, junto con la LST, algunos índices que afectan a la UHI, como el NDVI o el NDBI. En particular, el NDVI ayuda a conocer el efecto de la canopia verde y la calidad de la vegetación en la UHI de la RMB, así como de los casos de estudio en el SCV.

2. Análisis in situ

a. La información anterior se completará mediante la obtención de la LST día y noche in situ en los dos casos de estudio. Este segundo enfoque permitirá contrastar los resultados obtenidos a través de la teledetección. El mayor nivel de detalle de los datos de campo ayudará a ratificar la hipótesis de la investigación

b. Además, en los dos casos de estudio se tomarán mediciones de la LSAT de día y de noche. Esta información nos ayudará a confirmar la hipótesis del efecto microclimático del espacio público y de las infraestructuras verdes (tanto durante el día como durante la noche) desde la perspectiva de la percepción humana.

c. Finalmente, se analizará el diseño urbano de ambos casos de estudio y su infraestructura verde.

3. . Obtención de la LST

a. El método utilizado para obtener el LST utilizando información de LANDSAT consiste en convertir la codificación numérica (Número Digital-DN) de la banda térmica (calor infrarrojo) facilitada por el satélite en unidades físicas, es decir, en grados Celsius. Como tal:

b. EI DN se transforma en luminosidad espectral.

c. Se calcula la temperatura de brillo en el sensor. Esta temperatura no tiene en cuenta el tipo de material o tierra que emite la energía capturada, por lo tanto, sería equivalente a la temperatura emitida por un cuerpo negro.

d. El paso final para obtener el LST es corregir el valor numérico obtenido en los pasos anteriores, introduciendo la emisividad de los materiales del suelo. En

Citación: ARELLANO, B. y ROCA, J. Áreas verdes e Isla de Calor Urbana. En: Libro de proceedings, CTV 2018. XII Congreso Internacional Ciudad y Territorio Virtual. "Ciudades y Territorios Inteligentes". Mendoza, 5-7 septiembre 2018. Barcelona: CPSV, 2018, p. 417-432. 
este caso, la emisividad se ha obtenido del NDVI (índice de vegetación de diferencia normalizada). El uso del NDVI con respecto a otras alternativas, como la clasificación del uso del suelo tiene dos ventajas principales: 1. ambos, el índice de temperatura y vegetación provienen del mismo momento, y 2. la inmediatez involucrada en el cálculo de este índice.

La imagen utilizada (LC81970312015176LGN00) fue del verano de 2015 (un año extremadamente caluroso). Específicamente del 25 de junio. Imagen de LANDSAT 8 con las siguientes bandas: $1=$ Costera; $2=\operatorname{Azul}(30 \mathrm{~m}) ; 3=$ Verde $(30 \mathrm{~m}) ; 4=$ Rojo $(30 \mathrm{~m}) ; 5=$ Primer plano de infrarrojos $(30 \mathrm{~m}) ; 6=\operatorname{SWIR} 1(30 \mathrm{~m}) ; 7=\operatorname{SWIR} 2(30 \mathrm{~m}) ; 8=\operatorname{Pan}(15 \mathrm{~m}) ; 9$ = Cirrus; $10=\operatorname{TIR} 1(100 \mathrm{~m}) ; 11=\operatorname{TIR} 2(100 \mathrm{~m})$.

Figura 5. Bandas de Landsat

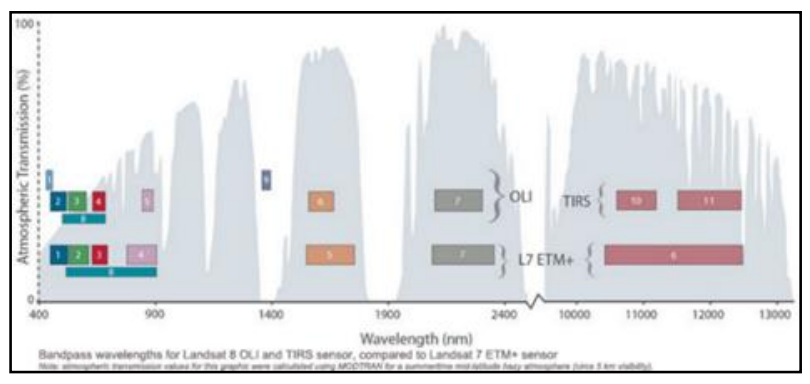

Figura 6. Proceso para la obtención de la LST

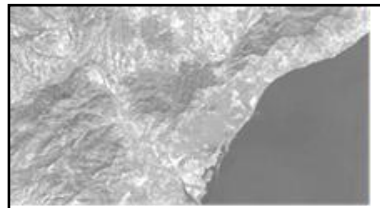

Digital Number

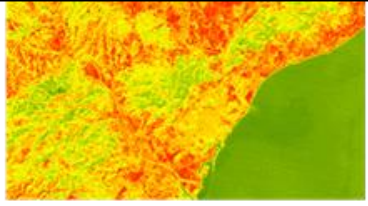

At sensor brightness Temp.

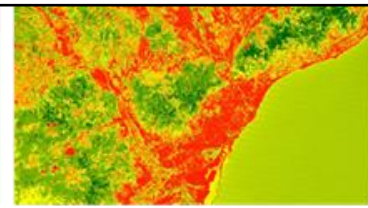

NDVI

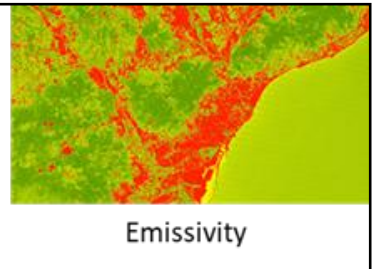

Emissivity

Figura 7. LST para la Región Metropolitana de Barcelona

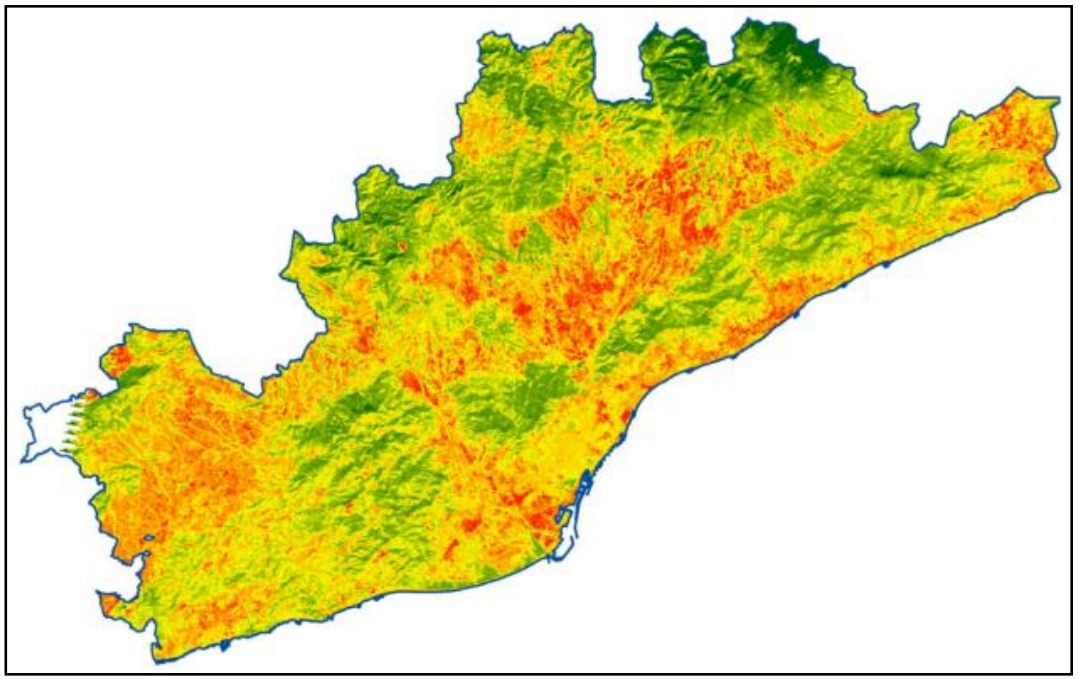

Fuente: Elaboración propia a partir de Landsat

Citación: ARELLANO, B. y ROCA, J. Áreas verdes e Isla de Calor Urbana. En: Libro de proceedings, CTV 2018. XII Congreso Internacional Ciudad y Territorio Virtual. "Ciudades y Territorios Inteligentes". Mendoza, 5-7 septiembre 2018. Barcelona: CPSV, 2018, p. 417-432. 


\section{Estudios de caso}

Los casos estudiados en esta investigación son los sectores de planificación de:

1. Parc Central (figura 8) y 2: Coll Favà (figura 9), ambos ubicados en el municipio de Sant Cugat del Vallés (a $10 \mathrm{~km}$ de Barcelona).

Al hacer la comparación entre ambas áreas de planificación, la mayoría de los indicadores de desarrollo tradicionales eran superiores en Coll Favà, y por tanto podría tener en principio un mejor comportamiento climático.

- La edificabilidad en Coll Favà $(0,61)$ es más bajo que en Parc Central $(0,85)$.

- Los espacios abiertos (sistemas) tienen una superficie mayor también en Coll Favà $(65.87 \%$ con respecto al $59.27 \%$ ).

- El porcentaje de espacios abiertos (viales y áreas verdes) también es mayor en Coll Favà (59.21\%) que en Parc Central (53.36\%).

- Finalmente, el porcentaje de suelo permeable es de $53.99 \%$ en Coll Favà y de $37.26 \%$ en Parc Central.

\section{Figura 8. Características generales de Parc Central}

\section{Planning area of 32.47 hectares, \\ with a private constructed area of $277,447 \mathrm{~m}^{2}$, \\ which represents $0.85 \mathrm{~m}^{2}$ of built up area for each $\mathrm{m}^{2}$ of land}

The public spaces (street network, urban facilities and infrastructures) covers $192,449 \mathrm{~m}^{2}, 59.27 \%$ of the area
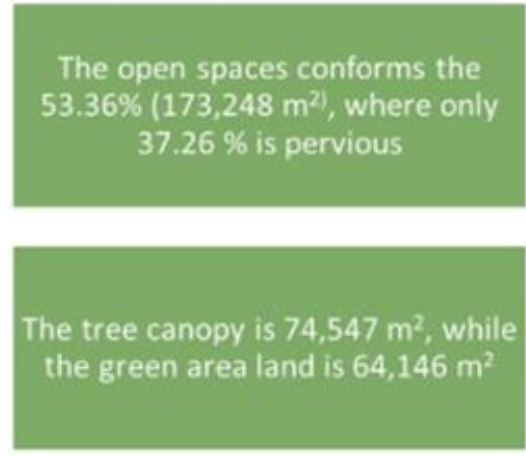

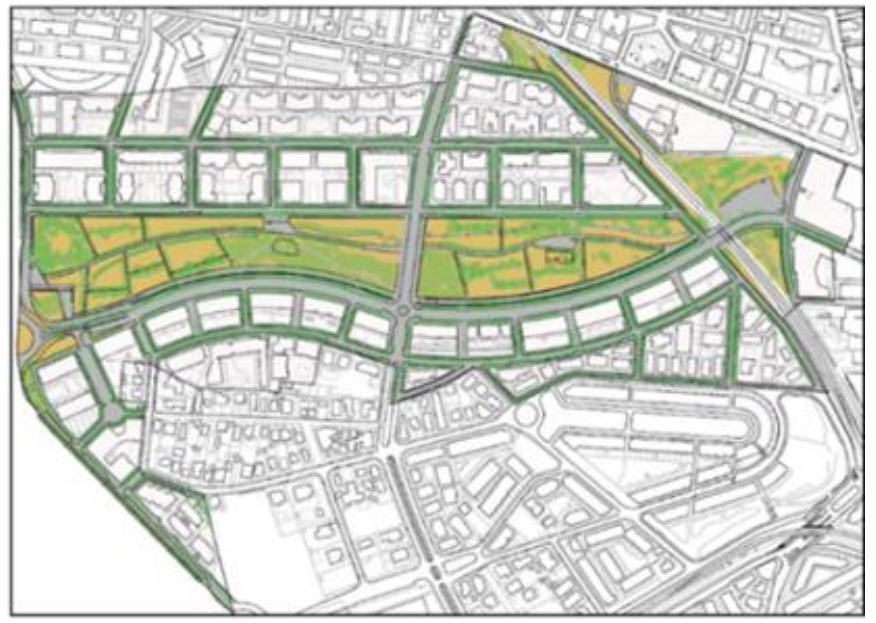

Citación: ARELLANO, B. y ROCA, J. Áreas verdes e Isla de Calor Urbana. En: Libro de proceedings, CTV 2018. XII Congreso Internacional Ciudad y Territorio Virtual. "Ciudades y Territorios Inteligentes". Mendoza, 5-7 septiembre 2018. Barcelona: CPSV, 2018, p. 417-432. 
Fig. 9. Características generales de Coll Favà
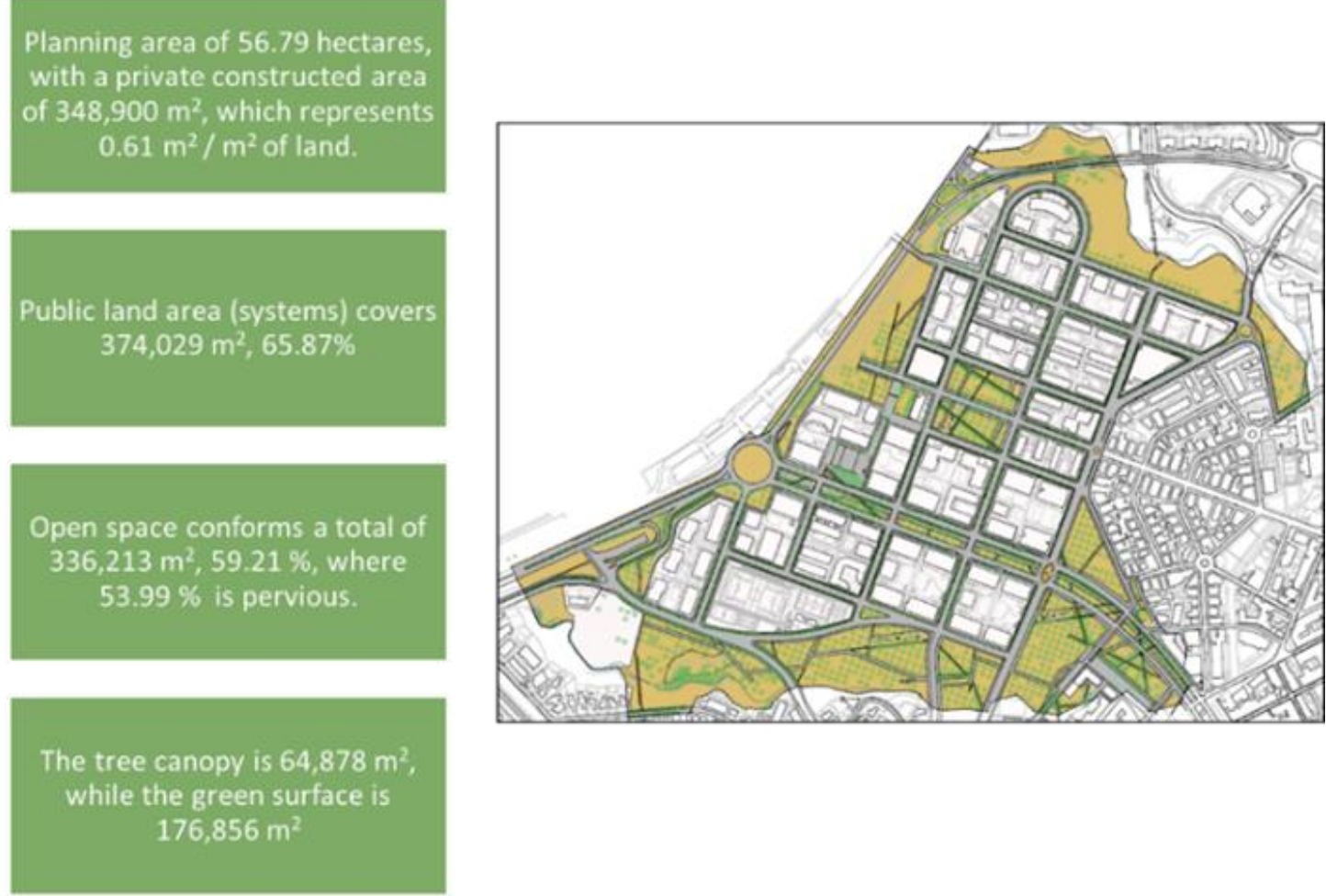

Desde un punto de vista urbanístico, la gran mayoría de los indicadores (tabla 1) sugiere un mejor desempeño climático de Coll Favà: menor área construida, mayor proporción de espacios públicos y abiertos y un mayor porcetaje de superficie permeable. Sólo un indicador (el dosel del árbol) favorece a Parc Central.

Tabla 1. Indicadores Urbanos

\begin{tabular}{|c|c|c|c|c|c|c|c|c|c|c|c|c|c|c|c|c|}
\hline & Superficie & $\begin{array}{c}\text { Suelo } \\
\text { público }\end{array}$ & \begin{tabular}{|c|} 
Suelo \\
privado
\end{tabular} & \% Público & \begin{tabular}{|l} 
Sup. \\
Construida
\end{tabular} & $\begin{array}{l}\text { Edif. } \\
\text { Bruta }\end{array}$ & $\begin{array}{l}\text { Edif. } \\
\text { Neta }\end{array}$ & Equip. & \begin{tabular}{|l} 
Espacio \\
Abierto
\end{tabular} & \begin{tabular}{|l} 
\% Espacio \\
abierto
\end{tabular} & Permeable & Impermeable & $\begin{array}{c}\% \\
\text { Permeable }\end{array}$ & \begin{tabular}{|c|} 
Sup.Verde \\
sobre \\
rasante
\end{tabular} & \begin{tabular}{|c|}
$\begin{array}{c}\text { Sup. Verde } \\
\text { bajo } \\
\text { rasante }\end{array}$ \\
\end{tabular} & Ratio \\
\hline & 696 & 192.449 & \begin{tabular}{|l|}
132.247 \\
\end{tabular} & 0,59 & 277.447 & 0,85 & 2,10 & 19.201 & 48 & 0,53 & 46 & \begin{tabular}{l|l|}
108.702 \\
\end{tabular} & 0,37 & 74.547 & 64.146 & 1621 \\
\hline coll Favà & 567.861 & 374.029 & 193.832 & 0,66 & 348.900 & 0,61 & 1,80 & 37.816 & \begin{tabular}{|l|}
336.213 \\
\end{tabular} & 0,59 & 181.512 & 154.701 & 0,54 & 64.878 & \begin{tabular}{|c|}
176.856 \\
\end{tabular} & 0,3668 \\
\hline
\end{tabular}

A pesar de sus peores "indicadores urbanos" tradicionales, podemos decir que Parc Central experimenta un mejor comportamiento térmico debido a la concentración compacidad del verde concentrado en sus áreas verdes. Al contrario, Coll Favà muestra una estructura más fragmentada de las zonas verdes. Parc Central también se ve beneficiado probablemente de un mejor microclima, debido a la mayor densidad de la canopia verde, a diferencia de Coll Favà, que es mucho más pobre y desnudo.

Esta hipótesis del mejor desempeño climático de Parc Central se ve confirmada por los siguientes indicadores:

- El análisis del NDVI revela una estructura muy diferente entre Coll Favà y Parc Central. Mientras que en la primera zona las áreas con vegetación densa son marginales, ocupando los límites del perímetro al este y al oeste de la zona, Parc Central muestra abundante vegetación en el centro de la zona.

Citación: ARELLANO, B. y ROCA, J. Áreas verdes e Isla de Calor Urbana. En: Libro de proceedings, CTV 2018. XII Congreso Internacional Ciudad y Territorio Virtual. "Ciudades y Territorios Inteligentes". Mendoza, 5-7 septiembre 2018. Barcelona: CPSV, 2018, p. 417-432. 
- El NDVI promedio en Coll Favà es de 0.3435, que es menor que el de Parc Central, que es 0.4529 . Esto significa que Parc Central tiene un promedio de calidad de vegetación mucho más alto que Coll Favà.

- Parc Central tiene un NDVI cercano al promedio municipal, que incluye casi el $50 \%$ de espacios naturales protegidos, como es el Parque Natural de Collserola.

- El análisis del NDVI a escala detallada (IGCC) confirma (ver figura 10) la calidad altamente diferenciada de la vegetación en ambas áreas.

- La vegetación por encima de la rasante (canopia verde) en Parc Central juega un papel fundamental, a diferencia de Coll Favà, donde las áreas "verdes" no tienen un follaje denso en la mayor parte del área, incluido el eje central del barrio.

\section{Figura 10. NDVI}

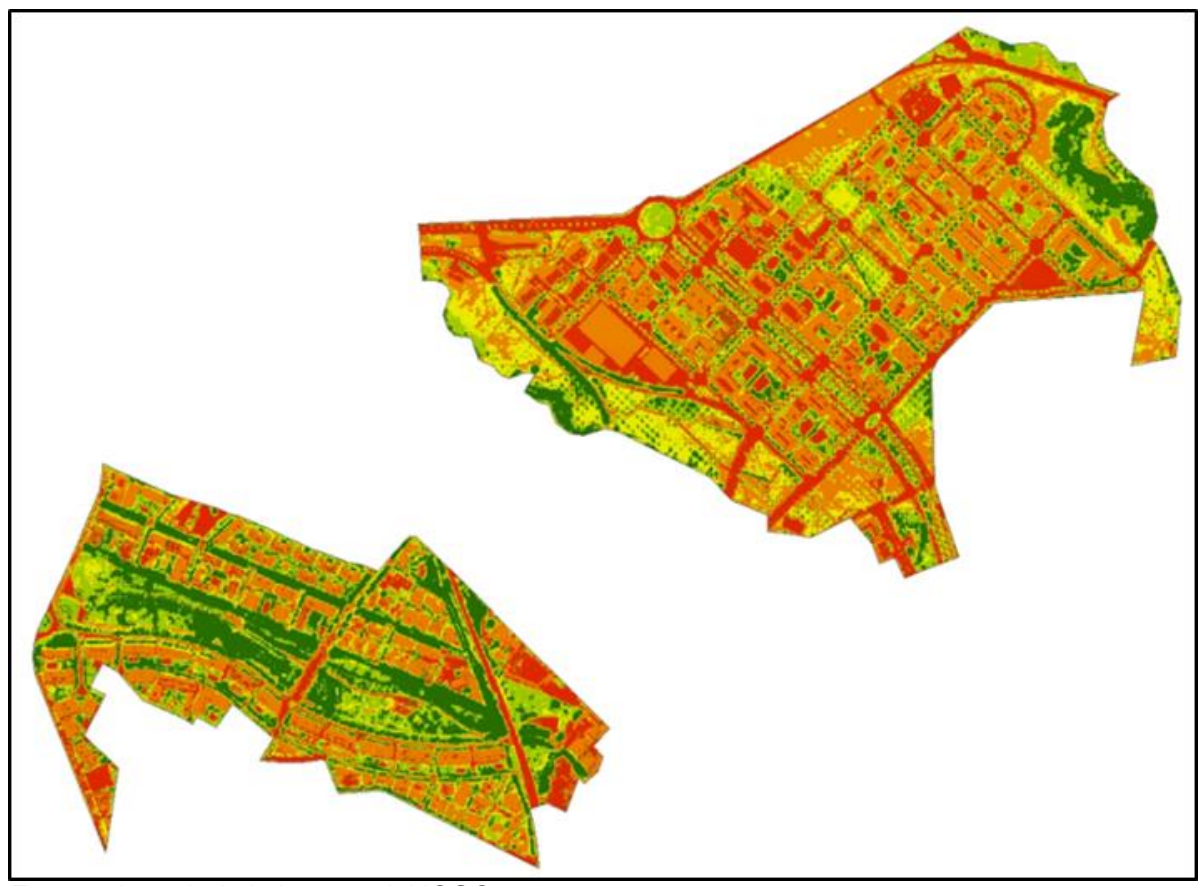

Fuente: A partir de la imagen del ICGC

El análisis de LST de la imagen de LANDSAT 8 confirma el mejor desempeño climático de Parc Central, con un promedio de $32.65^{\circ} \mathrm{C}$ en comparación con Coll Favà, con un LST promedio de $35.54^{\circ} \mathrm{C}$. Esta diferencia de casi 3 grados es altamente significativa a nivel intraurbano y no parece ser el resultado de factores geográficos diferenciales, dada la proximidad de las dos áreas. Los histogramas de la figura 11 sobre la LST para Parc Central y Coll Favà confirman los diferentes resultados climáticos de ambas áreas. La distribución de LST en Parc Central (azul) está claramente inclinada hacia la izquierda, es decir, hacia temperaturas más bajas. Coll Favà (verde), sin embargo, muestra una estructura clara, con un sesgo a la derecha. El análisis de los promedios $\left(32.53^{\circ} \mathrm{C}\right.$ PC y $35.44^{\circ} \mathrm{C} \mathrm{CF}$ ) y las medianas $\left(32.55^{\circ} \mathrm{C}\right.$ y $35.48^{\circ} \mathrm{C}$ respectivamente) confirma el diagnóstico.

Citación: ARELLANO, B. y ROCA, J. Áreas verdes e Isla de Calor Urbana. En: Libro de proceedings, CTV 2018. XII Congreso Internacional Ciudad y Territorio Virtual. "Ciudades y Territorios Inteligentes". Mendoza, 5-7 septiembre 2018. Barcelona: CPSV, 2018, p. 417-432. 
Figura 11. Histogramas de la LST de Parc Central (azul) y Coll Favà (verde)

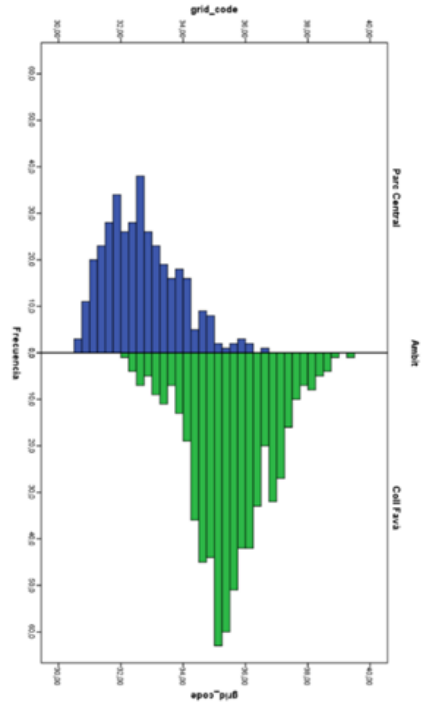

Si segmentamos las temperaturas de superficie terrestre según el tipo de espacio, tanto público como privado, los resultados son notablemente similares a los encontrados en todas las áreas de Parc Central y Coll Favà. Los espacios públicos del Parc Central tienen una temperatura promedio de $32.32^{\circ} \mathrm{C}$, en comparación con $35.64^{\circ} \mathrm{C}$ en Coll Favà. Si echamos un vistazo solo a terrenos privados, Parc Central obtiene un LST promedio de $33.15^{\circ} \mathrm{C}$, que también está claramente por debajo de Coll Favà, a $35.25^{\circ} \mathrm{C}$. El mejor rendimiento térmico de $P C$ no se limita, por lo tanto, al espacio público, sino que también se irradia al resto del tejido urbano, afectando las áreas privadas de una manera singularmente significativa.

Particularmente significativo es el hecho de que los espacios públicos en Coll Favà tienen una LST promedio más alta que los terrenos privados, al contrario de lo que se esperaría. Esto muestra el diseño inadecuado de los espacios verdes y abiertos en Coll Favà. Un ejemplo de esto es la zona deportiva "Jaume Tubau", cuya superficie de césped artificial hace una contribución negativa a la UHI en el área. Esto también es válido para áreas abiertas (permeables) sin vegetación o desnudas.

Además del análisis basado en la teledetección, se ha llevado a cabo un análisis "in situ" de la LST y la LSAT para verificar el comportamiento climático de Parc Central y Coll Favà. Por lo que se tomaron un conjunto de mediciones de campo con este propósito:

- Parc Central - Noche (noche del 2 al 3/09/16): se realizaron 19 imágenes térmicas, 4 videos y 20 mediciones de parámetros climáticos del aire.

- Parc Central - Día (mediodía, 3/09/16): 63 imágenes térmicas y 20 videos y 12 lecturas atmosféricas.

- Coll Favà - Día (mediodía, del 3 y 4/9/16): 31 imágenes térmicas, 13 vídeos y 12 lecturas atmosféricas.

- Coll Favà - Noche (noche 4/9/16): 53 imágenes térmicas, 16 videos y 12 lecturas atmosféricas.

Citación: ARELLANO, B. y ROCA, J. Áreas verdes e Isla de Calor Urbana. En: Libro de proceedings, CTV 2018. XII Congreso Internacional Ciudad y Territorio Virtual. "Ciudades y Territorios Inteligentes". Mendoza, 5-7 septiembre 2018. Barcelona: CPSV, 2018, p. 417-432. 
Fig. 12. LST in situ. Muestra de las imágenes térmicas
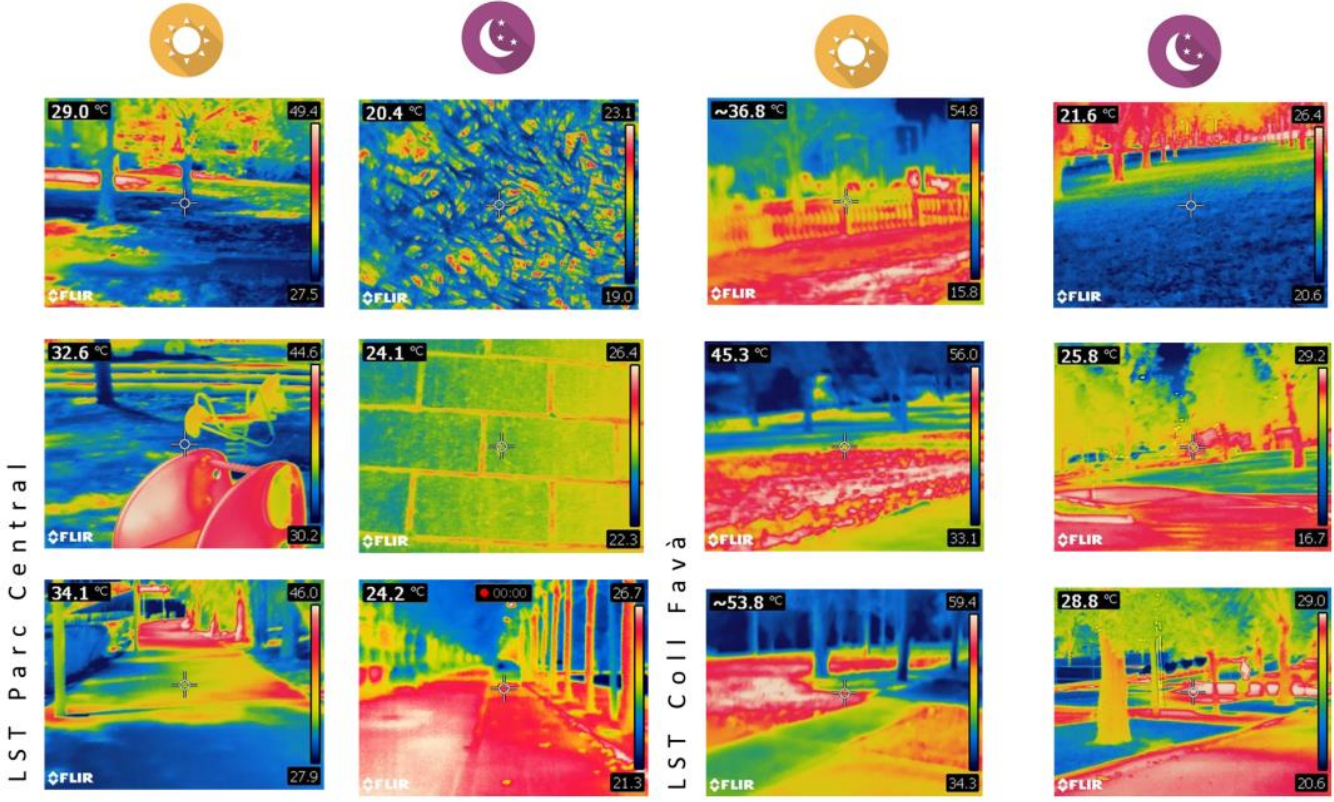

El análisis de las temperaturas diurnas de los pavimentos o las superficies de suelo confirma el mejor comportamiento térmico de Parc Central. Todas las superficies obtienen un LST inferior en Parc Central que en Coll Favà. La tierra desnuda obtiene mayores diferencias $\left(13.2^{\circ} \mathrm{C}\right)$, igual que el asfalto $\left(10.3^{\circ} \mathrm{C}\right)$. Las áreas pavimentadas en los parques se encuentran en un nivel intermedio $\left(8.5^{\circ} \mathrm{C}\right)$, las baldosas de terracota y bordes de acera $\left(8.2^{\circ} \mathrm{C}\right)$. Las superficies de césped presentan una diferencia térmica más pequeña $\left(4.7^{\circ} \mathrm{C}\right.$ más fríos en Parc Central que en Coll Favà). Asimismo, el análisis de las temperaturas nocturnas en las superficies del suelo confirma las temperaturas más bajas de Parc Central en comparación con Coll Favà. El suelo desnudo es el tipo de superficie que revela una mayor diferencia de temperatura, mientras que el césped y el asfalto revelan un mayor nivel de similitud en ambas áreas (tabla 3).

Tabla 2. Temperaturas nocturnas de los materiales.

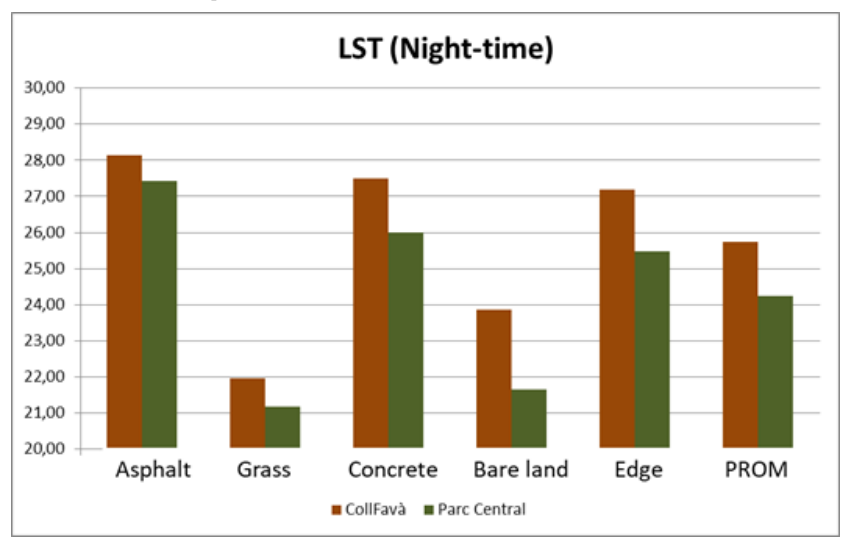

En conclusión, el análisis de las mediciones de la temperatura de superficie obtenida mediante el uso de la cámara térmica confirma ampliamente el mejor rendimiento de Parc Central:

Citación: ARELLANO, B. y ROCA, J. Áreas verdes e Isla de Calor Urbana. En: Libro de proceedings, CTV 2018. XII Congreso Internacional Ciudad y Territorio Virtual. "Ciudades y Territorios Inteligentes". Mendoza, 5-7 septiembre 2018. Barcelona: CPSV, 2018, p. 417-432. 
- El promedio para el grupo de mediciones obtenidas para la LST durante el día es de $34.08^{\circ} \mathrm{C}$ para el área del Parc Central, en comparación con los $44.30^{\circ} \mathrm{C}$ para Coll Favà: una diferencia de más de $10^{\circ} \mathrm{C}$.

- Por la noche, la lectura promedio del Parc Central es de $24.28^{\circ} \mathrm{C}$, en comparación con $24.91^{\circ} \mathrm{C}$ para Coll Favà. Las temperaturas tienden a ser iguales durante la noche, probablemente debido al enfriamiento significativo de las superficies permeables y libres de vegetación en Coll Favà.

- La temperatura media de la superficie disminuye en $9.8^{\circ} \mathrm{C}$ en Parc Central y en $19.39^{\circ} \mathrm{C}$ en Coll Favà.

Por lo tanto, Parc Central es mucho más fresco durante el día, aunque en la noche las temperaturas tienden a ser similares en ambas urbanizaciones.

Tabla 3. LST y LSAT

\begin{tabular}{|l|r|r|r|r|r|r|}
\hline & \multicolumn{3}{|c|}{ LST } & \multicolumn{3}{c|}{ LSAT } \\
\cline { 2 - 7 } & Diurna & Nocturna & \multicolumn{1}{c|}{ Dif. } & Diurna & Nocturna & \multicolumn{1}{c|}{ Dif. } \\
\hline Parc Central & 34.08 & 25.78 & 8.30 & 33.12 & 25.78 & 7.33 \\
\hline Coll Favà & 44.30 & 27.05 & 17.25 & 38.14 & 27.05 & 11.09 \\
\hline Diferencia & 10.22 & 1.27 & & 5.03 & 1.27 & \\
\hline
\end{tabular}

De la misma forma, el análisis de las temperaturas de aire (LSAT) in situ confirma las conclusiones anteriores:

- La temperatura promedio durante el día de Parc Central fue de $33.12^{\circ} \mathrm{C}, 5.03^{\circ} \mathrm{C}$ más baja que la de Coll Favà $\left(38.14^{\circ} \mathrm{C}\right)$.

- La temperatura nocturna promedio también es más fría en Parc Central $\left(25.78^{\circ} \mathrm{C}\right)$ que en Coll Favà $\left(27.05^{\circ} \mathrm{C}\right)$, aunque la diferencia se reduce a $1.27^{\circ} \mathrm{C}$.

- Esta reducción de las diferencias se debe principalente a las áreas de compensación o enfriamiento relativamente más altas en Coll Favà (especialmente en espacios descubiertos) con respecto a Parc Central: $11.09^{\circ} \mathrm{C}$ vs. $7.33^{\circ} \mathrm{C}$.

- Aun así, las mediciones nocturnas en Coll Favà superan las temperaturas máximas de confort $\left(24-26^{\circ} \mathrm{C}\right.$ grados).

El mejor comportamiento climático de Parc Central en comparación con Coll Favà es evidente en todos los análisis realizados. Tanto el estudio del LST diurno resultante de la teledetección, como las temperaturas superficiales del día y la noche resultantes del estudio de campo, muestran que el diseño de los espacios públicos y las áreas verdes del Parc Central ofrece un mejor rendimiento térmico que el Coll Favà. Resultados que también son confirmados por el análisis LSAT. 


\section{Conclusiones y recomendaciones}

Las principales conclusiones del trabajo se resumen a continuación:

1. A pesar de tener mejores "indicadores urbanos" tradicionales, Coll Favà observa un "comportamiento climático" más pobre que Parc Central.

2. El factor clave son las diferencias en las estructuras verdes. El dosel de los árboles, la canopia verde, es mejor en Parc Central que en Coll Favà. Además, es probable que Parc Central tenga un mayor alcance térmico debido a la mayor compacidad y concentración de la infraestructura verde que la fragmentación de Coll Favà.

3. El análisis del NDVI (Landsat 8) revela una estructura muy diferente entre Coll Favà y Parc Central. Mientras que en el primer barrio las áreas con vegetación densa son marginales, ocupando los límites del perímetro al este y al oeste de la zona, Parc Central muestra abundante vegetación en el núcleo central de la zona. Parc Central tiene un mejor microclima debido a la mayor densidad de la canopia verde, a diferencia de Coll Favà, con árboles muy altos y esbeltos y por tanto con un dosel mucho más pobre y desnudo.

4. El análisis de LST (LANDSAT 8) confirma el mejor desempeño climático de Parc Central, con un promedio de $32.65^{\circ} \mathrm{C}$ en comparación con Coll Favà, con un LST promedio de $35.54^{\circ} \mathrm{C}$.

5. El análisis "in situ" también confirma el mejor desempeño climático de Parc Central. Tanto de día como de noche. La LST y la LSAT son menores en Parc Central que en Coll Favà.

Podemos concluir que el uso de ambas tecnologías de análisis, sensores remotos y análisis "in situ" de las temperaturas de la superficie y del aire: han permitido una mejor identificación de la UHI. Como resultado de este estudio podemos afirmar que la morfología urbana juega un papel clave para generar y controlar la UHI; asimismo que la morfología urbana, el diseño del paisaje, la selección de la vegetación y de los materiales tienen especial relevancia en la resiliencia al cambio climático y en la resistencia a los eventos de ola de calor.

\section{Agradecimientos}

Los autores desean agradecer la ayuda recibida por el Gobierno de España (Ministerio de Economía y Competitividad, MINECO), así como a la Unión Europea (Fondo Europeo de Desarrollo Regional)

Citación: ARELLANO, B. y ROCA, J. Áreas verdes e Isla de Calor Urbana. En: Libro de proceedings, CTV 2018. XII Congreso Internacional Ciudad y Territorio Virtual. "Ciudades y Territorios Inteligentes". Mendoza, 5-7 septiembre 2018. Barcelona: CPSV, 2018, p. 417-432. 


\section{Bibliografía}

[1] Intergovernmental Panel on Climate Change, [Climate Change 2014. Synthesis Report], Contribution of Working Groups I, II and III to the Fifth Assessment Report of the Intergovernmental Panel on Climate Change, IPCC, Geneva, Switzerland, 151 pp.

[2] Trenberth, K.E., Jones, P.D., Ambenje, P., Bojariu, R., Easterling, D., Klein, T. A., Parker, D., Rahimzadeh, F., Renwick, J.A., Rusticucci, M., Soden, B. and Zhai, P., [Observations: Surface and atmospheric climate change. In Climate Change 2007: The Physics Science Basis], Cambridge University Press: Cambridge, New York, (2007).

[3] Weng, Q., "Thermal Infrared remote sensing for urban climate and environmental studies: Methods, applications, and trends", ISPRS Journal of Photogrammetry and Remote Sensing, Volume 64, Issue 4, July 2009, pag. 335-344 (2009).

[4] Zhou, W., Huang, G., Cadenasso, M. L., "Does spatial configuration matter? Understanding the effects of land cover pattern on land surface temperature in urban landscapes", Landscape and Urban Planning, Volume 102, Issue 1, 30 July 2011, pag. 54-63 (2011).

[5] Schwarz, N., Lautenbach, S., and Seppelt, R., "Exploring indicators for quantifying surface heat islands of European cities with MODIS land surface temperatures", Remote Sensing of Environment, 115, 3175-3186 (2011).

[6] US Environmental Protection Agency, "Reducing urban heat islands: Compendium of strategies. Draft", https://www.epa.gov/heat-islands/heat-island-compendium, (2008).

[7] Du, H., Cai, W., Xu, Y., Wang, Z., Wang, Y., and Cai, Y., "Quantifying the cool island effects of urban green spaces using remote sensing Data", Urban Forestry \& Urban Greening (27), 2431 (2017).

[8] Oliveira, S., and Andrade, H. V., "The cooling effect of green spaces as a contribution to the mitigation of urban heat: A case study in Lisbon", Building and Environment, 2186-2194 (2011).

[9] Arellano, B. and Roca, J., "Identifying Urban Heat Island at Medium Scale: The Barcelona Case", 11th Congress Virtual City and Territory, 11 VCT Proceedings book, 798-812 (2016).

Citación: ARELLANO, B. y ROCA, J. Áreas verdes e Isla de Calor Urbana. En: Libro de proceedings, CTV 2018. XII Congreso Internacional Ciudad y Territorio Virtual. "Ciudades y Territorios Inteligentes". Mendoza, 5-7 septiembre 2018. Barcelona: CPSV, 2018, p. 417-432. 